\title{
Cis-regulatory mutations associate with transcriptional and post-transcriptional deregulation of the gene regulatory program in cancers
}

Jaime A. Castro-Mondragon ${ }^{1}$, Miriam Ragle Aure ${ }^{2,3}$, Ole Christian Lingjærde ${ }^{2,4,5}$, Anita Langerød $^{2}$, John W. M. Martens ${ }^{6}$, Anne-Lise Børresen-Dale ${ }^{2}$, Vessela Kristensen ${ }^{2,3}$, Anthony Mathelier $1,2,3,{ }^{*}$

${ }^{1}$ Centre for Molecular Medicine Norway (NCMM), Nordic EMBL Partnership, University of Oslo, 0318 Oslo, Norway

${ }^{2}$ Department of Cancer Genetics, Institute for Cancer Research, Oslo University Hospital Radiumhospitalet, 0310 Oslo, Norway

${ }^{3}$ Department of Medical Genetics, Institute of Clinical Medicine, University of Oslo and Oslo University Hospital, Oslo, Norway

${ }^{4}$ Centre for Bioinformatics, Department of Informatics, University of Oslo, Gaustadalléen 23 B N-0373, Oslo, Norway

${ }^{5} \mathrm{KG}$ Jebsen Centre for B-cell malignancies, Institute for Clinical Medicine, University of Oslo, Ullernchausseen $70 \mathrm{~N}$ 0372, Oslo, Norway

${ }^{6}$ Erasmus MC Cancer Institute and Cancer Genomics Netherlands, University Medical Center Rotterdam, Department of Medical Oncology, 3015GD Rotterdam, the Netherlands

"To whom correspondence should be addressed: anthony.mathelier@ncmm.uio.no

\section{ABSTRACT}

Background: Most cancer alterations occur in the noncoding portion of the human genome, which contains important regulatory regions acting as genetic switches to ensure gene expression occurs at correct times and intensities in correct tissues. However, large scale discovery of noncoding events altering the gene expression regulatory program has been limited to a few examples with high recurrence or high functional impact.

Results: We focused on transcription factor binding sites (TFBSs) that show similar mutation loads than what is observed in protein-coding exons. By combining cancer somatic mutations in TFBSs and expression data for protein-coding and miRNA genes, we evaluated the combined effects of transcriptional and post-transcriptional alteration on the dysregulation of the regulatory programs in cancer. The analysis of seven cancer cohorts culminated with the identification of protein-coding and miRNA genes linked to mutations at TFBSs that were associated with a cascading trans-effect deregulation on the cells' regulatory program. Our analyses of cisregulatory mutations associated with miRNAs recurrently predicted 17 miRNAs as pan-cancerassociated through deregulation of their target gene networks. Overall, our predictions were enriched for protein-coding and miRNA genes previously annotated as cancer drivers. Functional enrichment analyses highlighted that cis-regulatory mutations are associated with the dysregulation of key pathways associated with carcinogenesis

Conclusions: These pan-cancer results suggest that our method predicts cis-regulatory mutations related to the dysregulation of key gene regulatory networks in cancer patients. It highlights how the gene regulatory program is disrupted in cancer cells by combining transcriptional and post-transcriptional regulation of gene expression. 


\section{INTRODUCTION}

Dysregulation of the gene expression regulatory program in a cell is a hallmark of cancer. The often observed aberrant gene expression in cancer can be triggered by deregulation at any regulatory level (transcriptional and post-transcriptional) [1,2]. While a majority of studies have focused on somatic mutations lying within protein-coding regions, most alterations occur in the noncoding portion of the human genome, which contains cis-regulatory elements acting as genetic switches to ensure gene expression occurs at correct times and intensities in the correct cells and tissues [3]. Molecular alterations at these regions can alter the regulatory network of the cells, conferring oncogenic behaviours, which has been associated with clinical and histopathological features in cancer [3]. However, identification of noncoding cancer driver events at cis-regulatory regions has been limited to a few examples with high recurrence or high functional impact [3-7]. In recent work based on mutation recurrence along the human genome, the Pan-Cancer Analysis of Whole Genomes (PCAWG) consortium, claimed that patients harbour $\sim 4.6$ driver mutations. The PCAWG consortium estimated that driver point mutations in noncoding regions ( -1.2 per patient) were less frequent than driver point mutations in proteincoding genes ( 2.6 per patient) [8]. However, large scale discovery of noncoding drivers has been hindered by their low level of recurrence, the target size of functional elements, technical shortcomings, and their composite effect with small individual effect size on multiple regulatory regions, e.g. slightly altering, but not obliterating, protein-DNA interactions $[4,8]$. Further, while high-impact driver mutations are typically sought, medium-impact putative passengers can have an aggregated effect in tumorigenesis, beyond annotated driver events [9].

Gene expression is mainly regulated at the transcriptional level by the binding of transcription factors (TFs) to promoters (cis-regulatory regions surrounding genes' transcription start sites, TSSs) and enhancers (cis-regulatory regions distal to genes) at TF binding sites (TFBSs) $[10,11]$. Most of the studies that predict noncoding driver mutations in cis-regulatory regions rely on the identification of mutational hotspots, which are regions with higher mutation frequencies than expected by chance [8,12-18]. Other studies explore somatic mutations with potential effect on TF-DNA interactions [19-22] based on DNA sequence information alone, followed by in vitro experiments to confirm the potential impact of the predicted mutations on gene expression. Other studies directly combine somatic mutation data with gene expression information to evaluate the impact of the mutations in cancer samples. For instance, studies identified differential allele-specific expression of genes between cancer and normal cells to pinpoint causal cis-regulatory variations in breast cancers [23,24]. Mutations close to the TSSs of genes were shown to exert in-cis effect on the expression of the corresponding genes [25]. Another example is the xseq tool that associates mutations with changes in expression in gene networks [26]. The tool has been originally developed to predict mutations in protein-coding exons with trans-effect [26] and adapted to consider noncoding mutations associated with protein-coding genes in B cell lymphomas [27]. This methodology specifically assesses the trans-associations between mutations and gene network expression alteration in cancer samples through either exonic or cis-regulatory mutations linked to protein-coding genes [26,27].

At the post-transcriptional level, miRNAs control gene expression by acting as 'buffers' to induce translational repression and mRNA degradation [28,29]. miRNA biogenesis generally comprises 
three steps in mammals: transcription of a primary transcript (pri-miRNA) that can be several kilobases long, cleavage of the pri-miRNA into a precursor (pre-miRNA) of $\sim 70 \mathrm{bp}$, and cleavage of the precursor to produce mature miRNAs of $\sim 22 b p[29,30]$. The mature miRNA sequence is then loaded in the RNA-induced silencing complex to specifically target mRNAs for repression through base pair complementarity at the 3'UTR of mRNA targets. A miRNA sequence is predicted to target tens to hundreds of mRNAs [31]. The influence of miRNA-based regulation on mRNA translation is not an on/off system but rather an interplay between miRNA-binding site specificity and miRNA abundance [28,32]. Therefore, even small changes in miRNA abundance may have an effect on the expression of several direct targets but also other mRNAs through a cascading effect, potentially leading to dysregulation patterns observed in cancer. This observation, amongst others, suggests that miRNAs can act as cancer drivers [33,34].

Despite active research on post-transcriptional regulation and the identification of miRNAs and their targets [35], the understanding of miRNA transcriptional regulation is currently limited [30]. One obstacle was the lack of precise identification of pri-miRNA TSSs. The FANTOM5 consortium recently took advantage of the cap analysis of gene expression (CAGE) technology to identify pri-miRNA TSSs genome-wide from different cell types and tissues in human and mouse [36]. Given their short size and the fact that they are not recurrently mutated [8], we hypothesize that the driver potential of miRNAs in cancer could be driven by cis-regulatory mutations that alter their expression in cancers with downstream cascading effect on the gene regulatory program of the cells.

The recent availability of high-quality sets of direct TF-DNA interactions [37], miRNA TSS locations [36], somatic cancer mutations, and cancer cell expression data [38] provides an unprecedented opportunity to analyze alterations of gene regulatory programs in cancer by looking at both transcription and post-transcriptional levels of gene expression regulation. The PCAWG consortium stated that the community is facing a 'paucity' in the discovery of noncoding cancer drivers that could be shortened by analyzing larger sample datasets [8]. We hypothesize that focusing on regulatory variants within TFBSs associated with protein-coding and miRNA genes combined with gene expression data has the potential to pinpoint cisregulatory variants linked to the dysregulation of key gene regulatory networks in cancer patients.

In this study, we adapted the framework of the xseq tool to predict cis-regulatory somatic mutations associated with the dysregulation of gene networks by considering both proteincoding and miRNA genes. We predicted genes that are associated with cis-regulatory mutations and cascading trans-effects on the gene regulatory program alteration across seven cancer patient cohorts from The Cancer Genome Atlas (TCGA) [38]. This analysis revealed 17 miRNAs recurrently predicted in the different cohorts. Functional enrichment analyses of the deregulated networks confirmed that pathways known to be associated with carcinogenesis are recurrently disrupted. We conclude that interpretation of noncoding mutations can be improved by focusing on TF-DNA interactions with the combined analysis of both transcriptional and posttranscriptional regulation of gene expression to revert the paucity in the discovery of cancerassociated noncoding events. 


\section{RESULTS}

Transcription factor binding sites harbour similar mutational load than protein-coding exons

We considered somatic mutations from whole genome sequencing of 349 samples from seven cancer patient cohorts (35 to 92 samples per cohort) covering seven distinct cancer types from TCGA [38] (Additional file 1). Specifically, we selected samples where trios of somatic mutations, RNA-seq, and small RNA-seq data were available. In aggregate, we examined $11,434,931$ somatic single nucleotide variants and small insertions and deletions (from 2,832 to 1,014,969 per sample; Additional file 2; Figure S1).

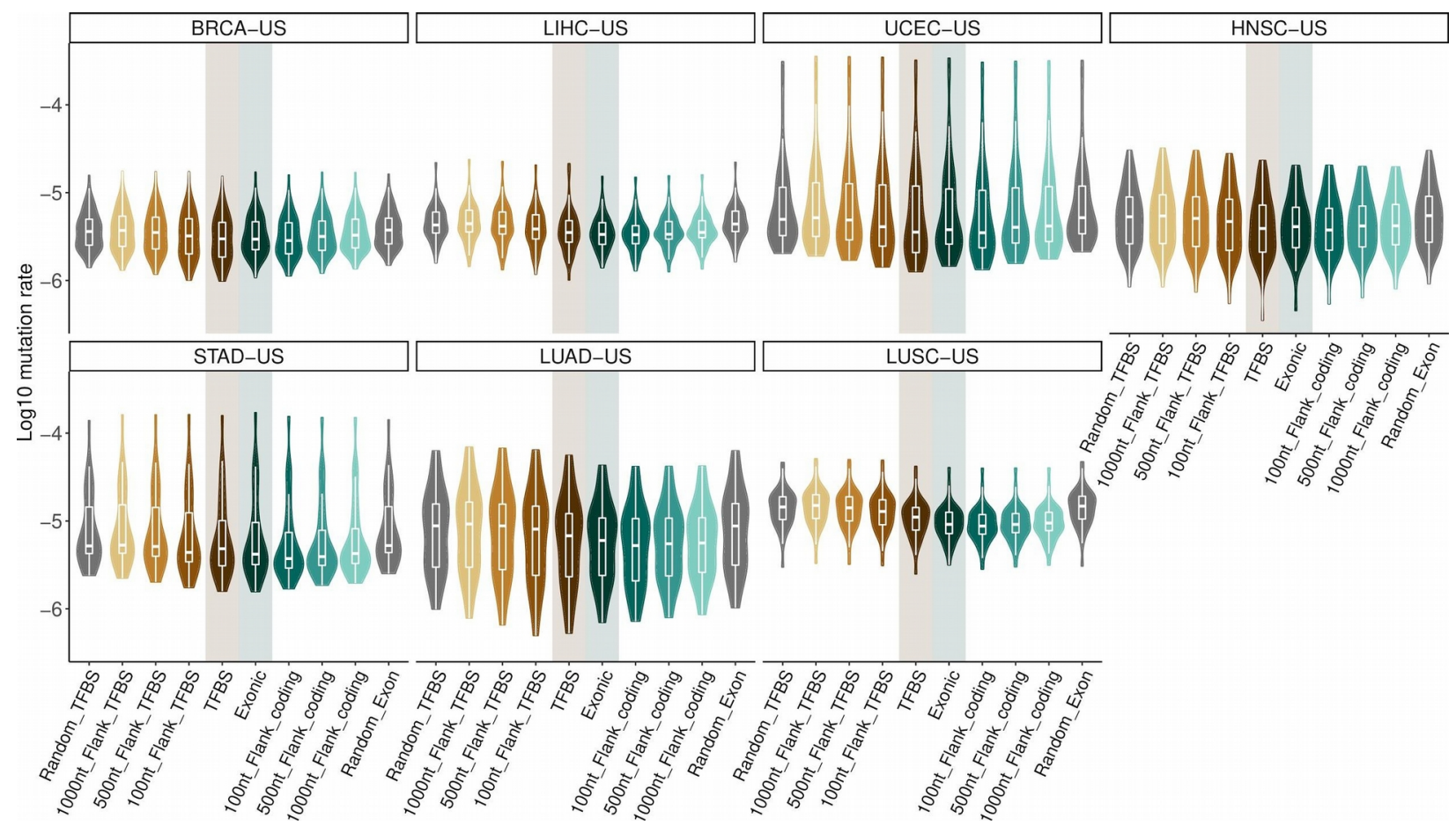

Figure 1. Comparison of mutation rates in TFBSs and exons versus their flanking regions and random mutation rates. Each panel corresponds to a specific cancer cohort (see title boxes) and each point corresponds to a sample. On each panel, the two central boxplots (shadowed) represent mutation rates in TFBS and exonic regions, the remaining box plots correspond to mutation rates in increasing-size flanking regions (100, 500, and 1000 nt) and mutation rates expected by chance (150 randomly distributed sets of mutations in the genome; Material and methods).

To highlight cancer-associated cis-regulatory mutations, we considered a set of TFBSs predicted as direct TF-DNA interactions in the human genome and stored in the UniBind database [37]. We first assessed whether this set of TFBSs would represent regions of functional interest similar to the coding portion of the human genome commonly studied to predict cancer-associated mutations. These TFBSs cover $\sim 2.2 \%(68,071,257 \mathrm{nt})$ of the human genome, close to the exonic coverage of protein-coding genes ( 2.6\%; 81,416,464 nt). Focusing on the somatic mutations, we observed that 1-2\% of the mutations in each sample are lying within these TFBSs (median of 277 mutations per sample; Additional file 2; Figure S2). As expected, mutation rates in TFBSs varied between cancer cohorts but were similar to the 
mutation rates observed in exons of protein-coding genes (two-tailed Wilcoxon tests p-values between 0.13 and 0.96; Figures 1 and S3-S4). We observed that TFBSs were less mutated than their surrounding sequences with mutation rates increasing as the size of the flanking regions increases (Figure 1 ). Note that regions of $1 \mathrm{~kb}$ surrounding TFBSs harbour mutation rates similar to what is expected by chance (two-tailed Wilcoxon tests p-values between 0.56 and 0.95; Figures S3-S4). While exons exhibit mutation rates similar to those observed within TFBSs (Figure S5), their flanking regions show a smaller increase in mutation rates than the increase detected in the vicinity of TFBSs (Figures S6-S7).

Taken together, these results highlight that the mutation frequencies in the studied set of TFBSs follow a similar pattern to what is observed in protein-coding exons. It provides an a posteriori confirmation that the set of TFBSs we considered is likely composed of functional regions in the human genome and could be used to highlight cis-regulatory mutations of functional interest in cancer genomes.

\section{Cis-regulatory and loss-of-function mutations are complementary mechanisms to alter protein-coding gene networks}

We sought to predict the cis-regulatory mutations lying in these TFBSs and that were linked to cascading effects on gene network deregulation, a hallmark of carcinogenic events. We first focused on the mutations linked to protein-coding genes and compared their effect to mutations altering the function of protein-coding genes. Specifically, we considered a protein-coding gene to be mutated through either a loss-of-function (LoF) somatic mutation in one of its exons as in ref. [26] or a somatic mutation overlapping a TFBS associated with the gene. TFBSs were linked to protein-coding or miRNA genes based on cis-regulatory element-to-gene associations from GeneHancer [39] or distances to TSSs (Material and methods; Figure S8). We related the mutations to their potential trans-effect on expression disruption in protein-coding gene networks using the xseq tool, following approaches implemented in previous studies [26,27]. Specifically, the method uses a hierarchical bayesian approach to associate mutations with expression dysregulation in biological networks associated with the mutated protein-coding genes. In a nutshell, it assesses the posterior probability of the likely association between observing mutations in a set of patients and observed deviations from neutral expression in these samples for protein coding genes in the same network. The likely trans-associations between mutations and gene network deregulation are first assessed in a sample-specific manner and then across samples from the same cohort (Figure S9). Genes with low expression in a given cohort were filtered out; the distribution of the 90th percentile of expression for genes was decomposed into two Gaussian distributions corresponding to low and high expression values and only genes lying in the high expression distribution were conserved (Material and methods). Further, we corrected for copy number alteration to compensate for their cis-effect on expression (Material and methods). LoF mutations and mutations overlapping TFBSs were analyzed independently.

Pan-cancer analyses of the seven TCGA cohorts predicted 30 protein-coding genes when considering LoF mutations (none in HNSC-US, LUAD-US, and LUSC-US; 2 in LIHC-US; 4 in BRCA-US; 9 in STAD-US; 18 in UCEC-US) and 283 genes when considering cis-regulatory 
mutations (6 in LIHC-US; 22 in BRCA-US and HNSC-US; 35 in LUSC-US; 42 in STAD-US; 81 in LUAD-US; 107 in UCEC-US) (Figures 2 and S10-S12). Three genes were linked to dysregulated networks in association with both LoF and cis-regulatory mutations but in different patients and cohorts: ACVR2A, ARID1A, and GATA3 (Figure 2A). These three genes are already known cancer drivers that we predict to be impacted by alternative mutational mechanisms (LoF or cis-regulatory mutations). For the other genes, we observed that they were either associated with LoF mutations or mutations in TFBSs across cohorts (e.g. TP53, RPL22, and PDS5B with LoF mutations; PIK3C3 and CEBPB with cis-regulatory mutations; Figure 2).

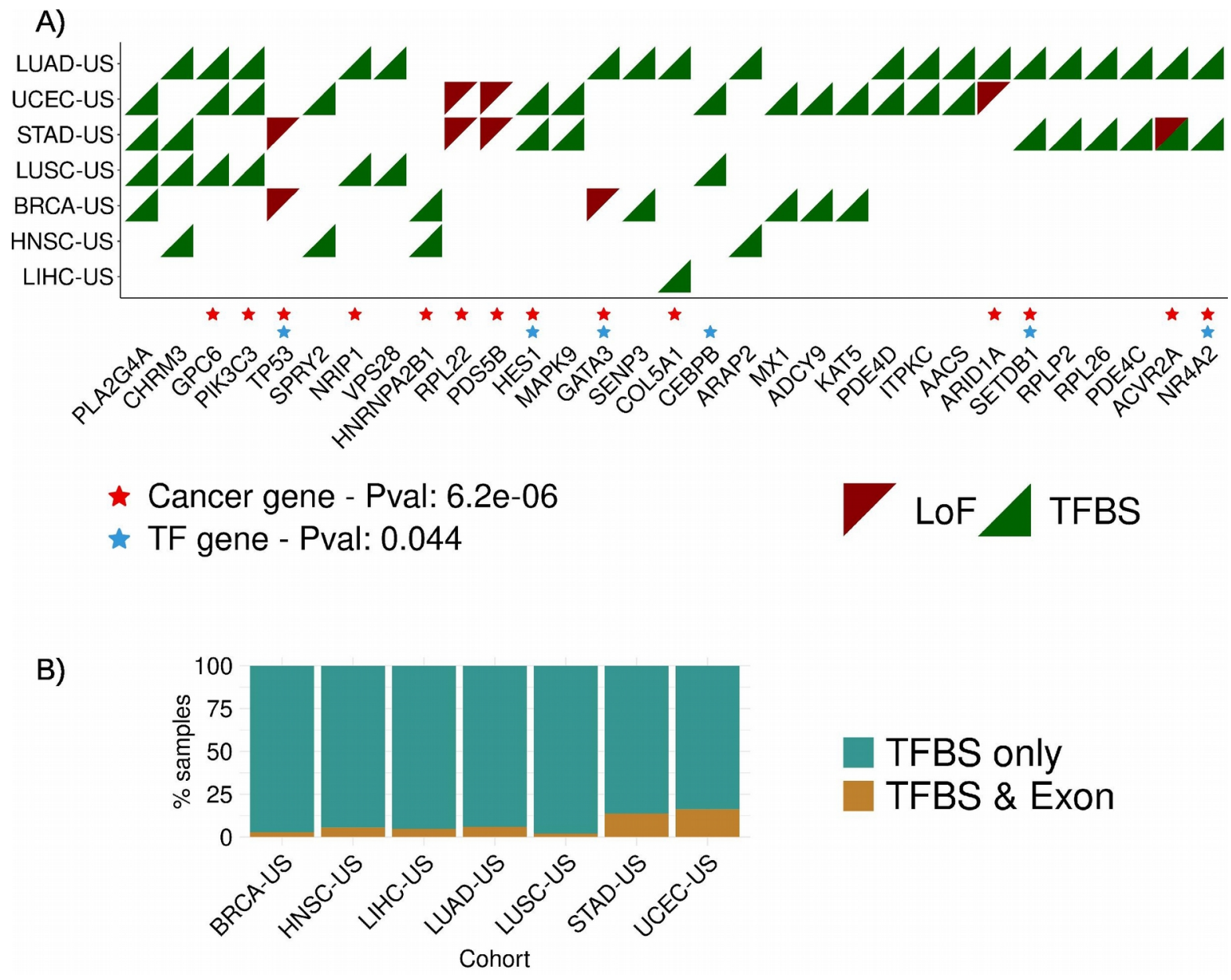

Figure 2. Pan-cancer predicted protein-coding genes. A) Predictions were obtained applying the $x$ seq tool when considering protein-coding genes mutated through either LoF (red triangles) or cis-regulatory (TFBS; green triangles) mutations, independently. Genes predicted in at least two cohorts are depicted here. Genes known as cancer genes (red stars) and TFs (blue stars) were found to be enriched (hypergeometric tests; $p$-values provided in the legend; Material and methods). B) Samples where genes were predicted through cis-regulatory mutations were considered for each cohort and assessed for the presence of LoF mutations in the same genes for the same cohort (TFBS \& Exon) or no LoF mutation in the corresponding gene (TFBS only).

From the combined list of 310 predicted protein-coding genes (Additional Files 3 and 4), 87 were already annotated as cancer genes ( $p$-value =1.5e-16; hypergeometric test) and 37 as TFs ( $p$-value $=0.0061$; Figures S10-S12). Considering recurrent predictions over cancer types, we observed 31 genes to be predicted in at least two cohorts. These 31 genes are enriched for already known cancer drivers ( $p$-value $=6.2 \mathrm{e}-6$; hypergeometric test) and TFs ( $p$-value = 0.044; 
hypergeometric test) (Figure 2A).

The genes identified through cis-regulatory mutations rarely contained LoF mutation in the same patients (Figures $2 \mathrm{~B}$ and $\mathrm{S} 13$ ). These results reinforce the possible complementary mechanisms between LoF and cis-regulatory mutations at play in cancer patients to alter the gene regulatory program of cancer cells. We observed that multiple genes could be predicted in the same sample through cis-regulatory mutations (e.g. from 1 to 42 genes were predicted in UCEC-US samples). Nevertheless, these genes tend to be interconnected in the dysregulated genes' networks with a maximum of 5 disconnected subgraphs per sample (Figure 3). All these genes are predicted through mutations associated with cascading trans-effect in gene network dysregulation but the method cannot pinpoint which specific event could be the main driver event or if it is due to the combination of cis-regulatory mutations. When considering all the predicted genes per cohort, we detect a similar pattern with subnetworks of interconnected genes (Figures 3B and S14). Altogether, these interconnections suggest that the predicted genes are likely involved in similar biological pathways with altered expression associated with cis-regulatory somatic mutations.
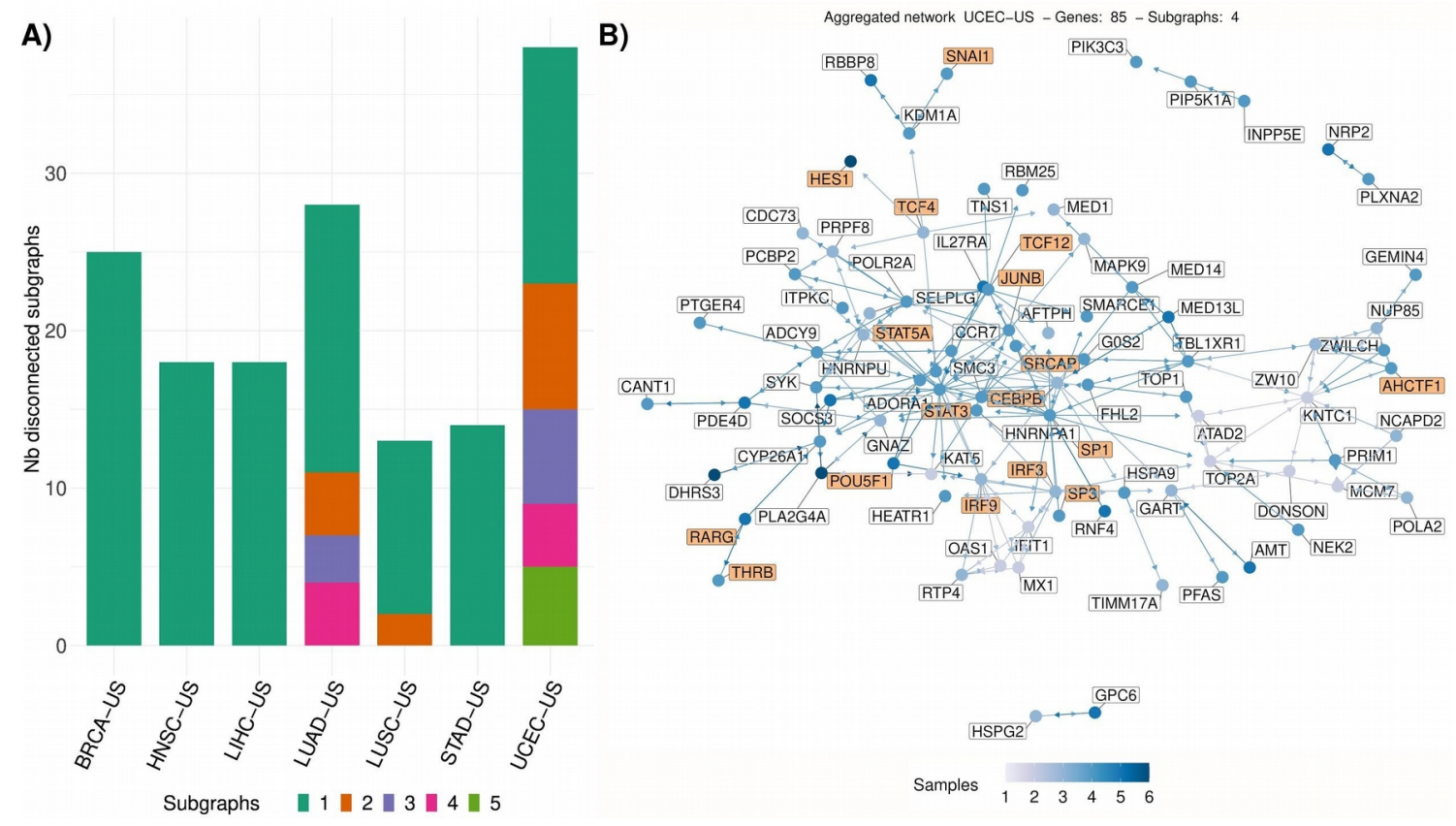

Figure 3. Networks of predicted protein-coding genes. A) Stacked histogram depicting the number of disconnected networks of predicted protein-coding genes (see legend) per sample (number of samples on the $y$-axis) for each cohort (x-axis). B) Network of all predicted genes in the UCEC-US cohort. The number of samples in which each gene was predicted is provided using a color scale (see legend). TF genes are highlighted with an orange background.

\section{Deregulation of transcriptional activity and cancer pathways are trans-effect signature of the predicted cis-regulatory and loss-of-function mutations}

Next, we performed enrichment analyses to shed light on the functional role of the somatic mutations predicted to be associated with a cascading effect on gene expression alteration. One advantage of $x s e q$ is its capacity to highlight the specific genes in the biological networks associated with the candidate cancer-associated genes that are dysregulated in the samples 
harbouring the somatic mutations considered (Material and methods) [26]. We observed that these genes were consistently found to be either up- or down-regulated in the samples with predicted disrupted expression (see the blue and red colors in the upper and lower clusters in Figure 4A). These results highlight sets of genes up- or down-regulated across samples where cancer-associated genes are predicted.

We assessed the biological relevance of the networks predicted to be dysregulated in association with the protein-coding genes predicted through LoF or cis-regulatory mutations. Functional enrichment analysis was performed using pathways from KEGG [40], WikiPathways [41], and Panther [42], and gene ontology biological processes (GO BP [43]) with the EnrichR tool [44]. The dysregulated genes in the networks are enriched for transcriptional activity ('regulation of transcription, DNA-templated' from GO BP; Figure S15). Combined with the enrichment of TFs in the list of predicted cancer-associated genes, this result emphasizes that the alteration of transcriptional regulation is a common feature of cancer cells throughout cancer types. Focusing on biological pathways enriched in our list of genes from the dysregulated networks, we found pathways already known to be associated with carcinogenesis at the top of the enriched terms (e.g. 'Pathways in cancer', 'WNT signaling', 'PI3K-Akt signaling', and 'Focal adhesion'; Figures 4B-C and S15-S18). These results confirm that our approach highlighted somatic exonic and cis-regulatory mutations associated with potential protein-coding cancer-associated genes with cascading effect on regulatory alteration of key cancer-related pathways.

The enrichment for cancer pathways represents a posteriori confirmation that our method can pinpoint somatic events likely associated with carcinogenesis. Nevertheless, our results suggest that alteration of gene network expression in different patients could be achieved through cisregulatory mutations associated with different genes involved in the same pathway.

\section{Combining transcriptional and post-transcriptional regulation highlights pan- cancer miRNAs associated with gene expression alteration}

The analysis of protein-coding genes presented above exhibited that our methodology is able to pinpoint cis-regulatory mutations likely associated with carcinogenesis. With miRNAs involved in post-transcriptional regulation of gene expression, we hypothesized that our method could highlight cis-regulatory mutations linked to miRNAs with downstream cascading effect on the gene regulatory program of the cells. This new analysis aimed at combining transcriptional (through mutations in TFBSs) and post-transcriptional (through miRNA-targets regulatory networks) regulation to predict miRNAs associated with a trans-effect on gene expression alteration through somatic mutations in cis-regulatory elements. 
bioRxiv preprint doi: https://doi.org/10.1101/2020.06.25.170738; this version posted June 26, 2020. The copyright holder for this preprint (which was not certified by peer review) is the author/funder, who has granted bioRxiv a license to display the preprint in perpetuity. It is made available under aCC-BY 4.0 International license.

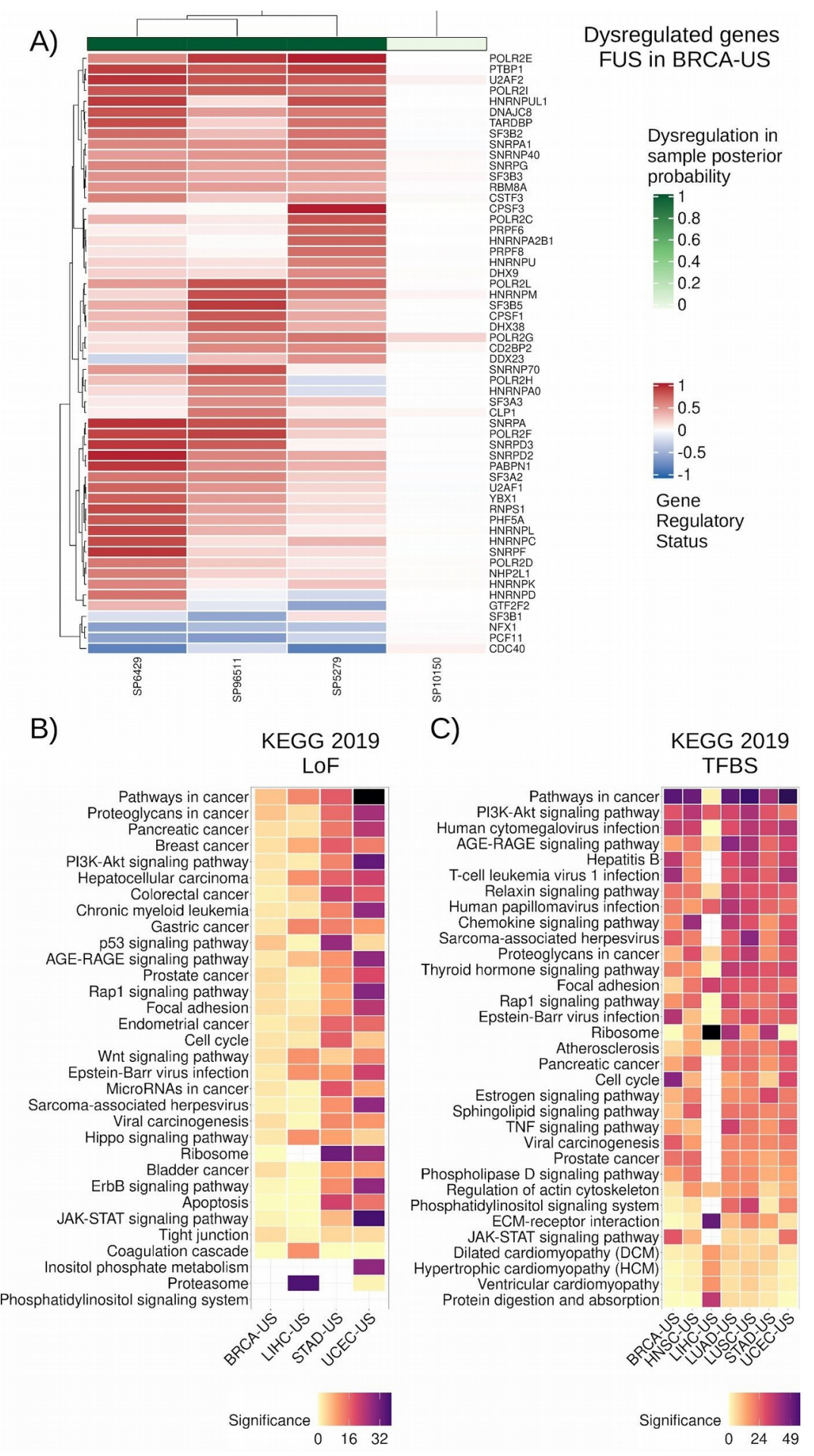

Figure 4. Dysregulated protein-coding gene networks and functional enrichment analysis. A) Dysregulated gene network in samples where FUS is predicted through cis-regulatory mutations in BRCA-US (rows: dysregulated genes associated with FUS; columns: samples with FUS-associated cis-regulatory mutations). The color scale represents the gene regulatory status posterior probability (red: up-regulation; blue: down-regulation). The green horizontal bar on top shows the sample-specific dysregulation posterior probability computed by xseq for the samples harboring a cis-regulatory mutation in the FUS gene. B) KEGG 2019 most enriched terms computed from all the dysregulated genes associated with the predicted protein-coding genes (Figure 4A is one example for GATA3) by xseq with LoF and C) cis-regulatory mutations in TCGA cohorts (columns). Terms (rows) are ordered by their mean rank across all cohorts. Significance is provided as $-\log _{10}(p-v a l u e)$. 
Pan-cancer analyses of the seven TCGA cohorts predicted 98 miRNAs derived from 63 premiRNAs as potential cancer-associated miRNAs (Figure S19; Additional Files 3 and 4). From these 98 miRNAs, 73 were already annotated as cancer miRNAs in the miRCancer database [48] ( $p$-value = 9.6e-28; hypergeometric test), which is derived from text-mining of the scientific literature in PubMed [49]. Moreover, miRCancer provides information about the cancer types that are associated with miRNAs in the literature; $\sim 35 \%$ of the predictions of cancer miRNAs in specific cancer types were supported by the literature to be involved in the same cancer type ( $p$ value $=7.02 \mathrm{e}-27$; hypergeometric test).

We identified a core set of 17 miRNAs (derived from 9 pre-miRNAs) that were identified in at least five out of the seven cohorts (Figure 5A): hsa-miR-17-3p, hsa-miR-17-5p, hsa-miR-20a-3p, hsa-miR-20a-5p, hsa-miR-708-3p, hsa-miR-708-5p, hsa-miR-92a-1-5p (predicted in all 7 cohorts), hsa-miR-18a-3p, hsa-miR-18a-5p, hsa-miR-155-3p, hsa-miR-155-5p (6 cohorts), hsamiR-205-3p, hsa-miR-205-5p, hsa-miR-324-3p, hsa-miR-324-5p, hsa-miR-629-3p, and hsamiR-629-3p (5 cohorts). We did not observe a correlation between the number of potential target genes for a miRNA and the number of cohorts where it is predicted (Figure S20). All these miRNAs are derived from precursors of already established oncomiRs or tumor suppressor miRNAs, or known to be involved in immune response or inflammation [50-61]. Note that hsa-miR-17-3p, hsa-miR-17-5p, hsa-miR-18a-3p, hsa-miR-18a-3p, hsa-miR-20a-3p, hsa-miR-20a-5p, and hsa-miR-92a-1-5p are part of a single miRNA cluster on chromosome 13 and this polycistronic cluster (known as miR-17-92) is well known to be composed of oncomiRs involved in proliferation and tumor angiogenesis, and reducing apoptosis of cancer cells [50].

When visualizing the dysregulated networks of miRNA targets in samples harbouring the predicted cancer-associated miRNAs, we observed subsets of the networks as up- or downregulated across patients from the same cohort (Figure 5B). This observation is similar to what we detected in protein-coding gene networks (Figure 4A). Note that the miRNA target networks observed with altered expression for a given miRNA may vary between cohorts for the same miRNA as some targets are specifically expressed or altered in a subset of tissues or cell types (Figure S21).

Similar to what we detected with disrupted gene networks of protein-coding genes, functional enrichment for miRNA targets with altered expression highlighted transcriptional activity terms and biological pathways associated with carcinogenesis (Figure 5C). Further, these results were recurrently found when considering disrupted target genes in each cohort independently (Figures S15-S18). We discovered several virus infection-related terms enriched across the cohorts (Figures 4B-C and 5C), arguing for a potential link between viral infections and cancer initiation/progression, as previously suggested [62,63], via miRNAs.

Altogether, this study provides a first foray in the analysis of a combined effect of transcriptional and post-transcriptional dysregulation downstream of somatic cis-regulatory mutations associated with miRNAs in cancer cells. It highlights a core set of miRNAs associated with cisregulatory mutations that are linked to a cascading alteration of gene regulatory networks involved in cancer onset and progression. 
A)

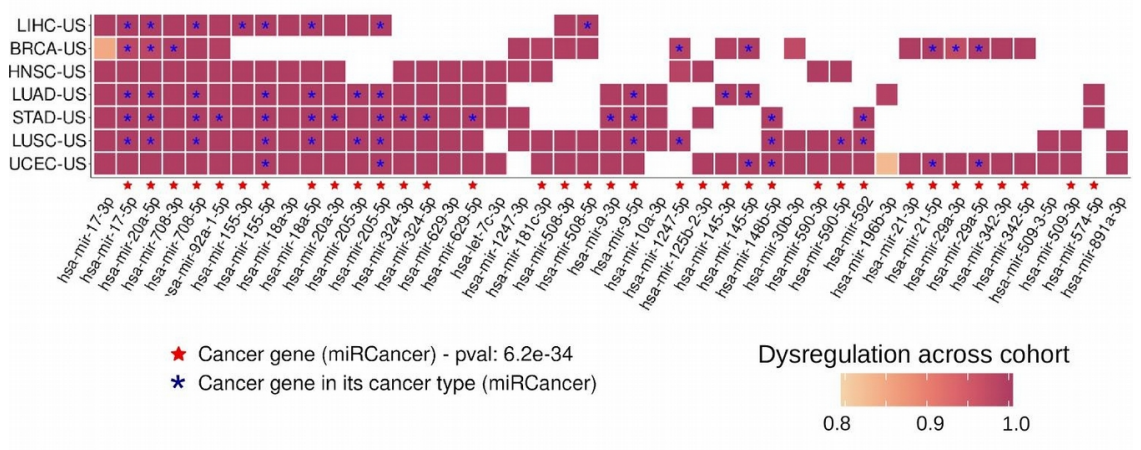

B) Dysregulated genes hsa-mir-29a-3p in BRCA-US

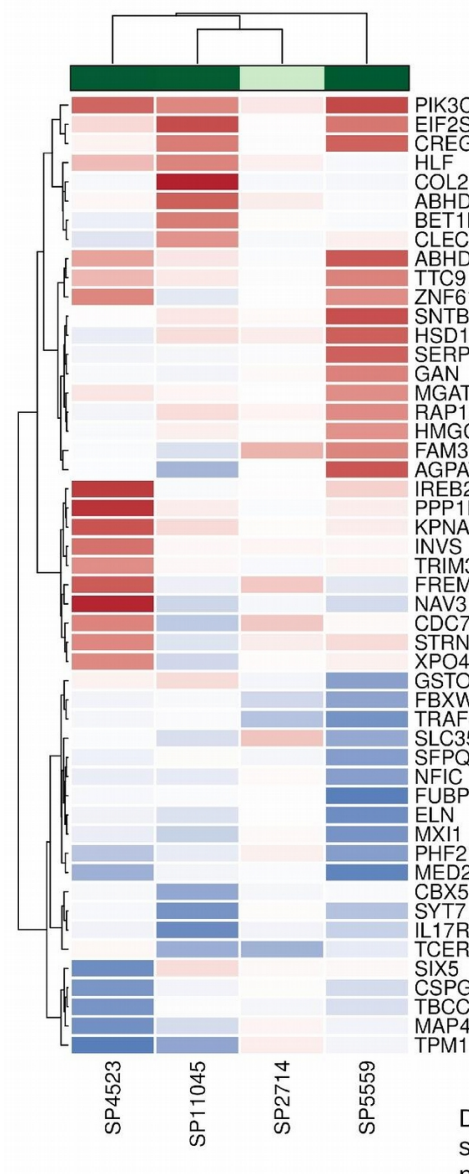

C)

KEGG 2019

TFBS associated to miRNAs

Proteoglycans in cancer

Pathways in cancer

Endocytosis

Colorectal cancer

MAPK signaling pathway

Pancreatic cancer

Hepatitis B

Hippo signaling pathway

Axon guidance

HIV 1 infection

Wnt signaling pathway

FoxO signaling pathway

Human cytomegalovirus infection

Ubiquitin mediated proteolysis

Hepatocellular carcinoma

Rap1 signaling pathway

AGE-RAGE signaling pathway

Sphingolipid signaling pathway

Thyroid hormone signaling pathway

Gastric cancer

Prolactin signaling pathway

Protein processing in ER

Fluid shear stress and atherosclerosis

\section{Gene}

Regulatory

Status

-1
0.5
0
-0.5
-1
-1
-0.8
-0.6
0.4
0.2
0

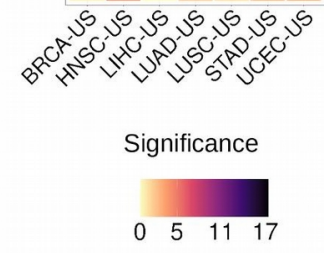

Dysregulation in sample poster
probability

Figure 5. Overview of miRNA driver predictions and their dysregulated target networks. A) miRNAs predicted as potential drivers by $x$ seq in at least two TCGA cohorts. Cell colors indicate the posterior probability computed over the corresponding cohort. Red stars indicate that the miRNA is annotated as a cancer miRNA in miRCancer [48]. Blue stars indicate that the miRNA was reported as a cancer miRNA in the specific cancer type where it is predicted by $x$ seq, according to miRCancer annotation. B) Dysregulated network of target genes for hsa-mir-29a-3p predicted in BRCA-US (rows: dysregulated targets; columns: samples with cis-regulatory mutations associated with hsa-mir29a-3p). The color scale represents gene regulatory status posterior probability (red: up-regulation; blue: downregulation represented as the posterior probability times -1). C) KEGG 2019 most enriched terms (rows) for all the dysregulated genes associated with the identified miRNA drivers across TCGA cohorts (columns). Terms are ordered by their mean rank across all cohorts. Significance is provided as $-\log _{10}(p-v a l u e)$. 


\section{Complementary analysis of independent breast cancer cohorts supports several cancer-associated miRNA predictions}

We aimed to assess the recurrence of the predictions for breast cancer obtained from the 92 samples of the BRCA-US cohort from TCGA in a complementary cohort. We applied the same methodology with the same parameters on the BASIS breast cancer cohort [64], which is composed of 256 breast cancer samples with the same trio of data types available (WGS, RNAseq, and miRNA expression - from microarrays; Additional file 5).

Similar to the BRCA-US TCGA analysis on protein-coding genes, our analysis of the BASIS cohort predicted known cancer drivers identified by associating LoF or cis-regulatory mutations with dysregulation of their gene networks. Further, we observed enrichment of similar key cancer pathways when considering the dysregulated genes associated with the predicted cancer-associated genes (Figures S22-S23). Breast cancers can be categorized into estrogen receptor positive $(E R+)$ and negative (ER-), each subtype harbouring a distinctive signature of gene expression. We explored how the distribution of ER status in patients from the two cohorts could impact the predictions of cancer-associated genes. The TCGA BRCA-US cohort is composed of approximately the same number of ER+ and ER-patients while the BASIS cohort is composed of $72 \%$ of ER+ patients. Given the size of the BASIS cohort (256 samples), it was possible to perform two additional analyses on ER+ (184 samples) and ER- samples (72 samples) independently. The analysis of cis-regulatory mutations associated with protein-coding genes revealed one prediction common to TCGA and ER+ BASIS cohorts (IL12RB1; Figure S24). Despite this small intersection, the functional enrichment analysis of the dysregulated genes associated to all predicted genes were similar in the two cohorts (Figures S25-S26), suggesting that although the predictions vary among cohorts with different aethiology, the dysregulated pathways are likely the same.

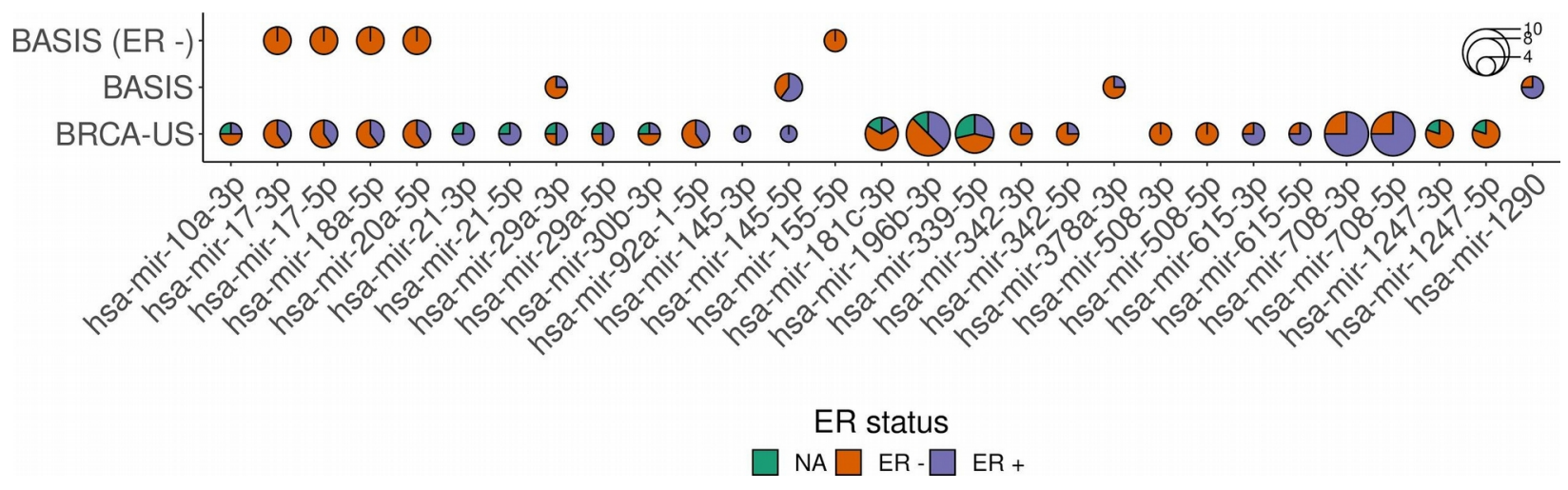

Figure 6. Predicted miRNA drivers in breast cancer cohorts. Predicted miRNAs in TCGA (BRCA-US) and BASIS (all samples and ER- samples only). The pie plots represent the distribution of samples with ER-/+ status.

We predicted four miRNAs associated with cis-regulatory mutations in the BASIS cohort when considering all samples (Figure 6). Two of these miRNAs, hsa-miR-145-5p and hsa-mir-29a-3p, were previously identified by our methodology using the BRCA-US cohort. We did not predict any driver miRNAs associated with cis-regulatory mutations when examining specifically the $\mathrm{ER}+$ samples. However, we identified hsa-mir-17-3p, hsa-mir-17-5p, hsa-mir-18a-5p, hsa-mir20a-5p, and hsa-mir-155-5p when considering ER- samples. These five miRNAs were 
recurrently found across multiple TCGA cohorts (Figures 5-6). Note that when predicted using the BRCA-US TCGA cohort, hsa-mir-17-3p, hsa-mir-17-5p, hsa-mir-18a-5p, and hsa-mir-20a-5p miRNAs were predicted in a majority of ER- samples as well. As expected, these results confirm that the cohort clinicopathological composition impacts the predictions as it can impact the landscape gene expression distributions across samples. Nevertheless, the complementary analyse of the TCGA and BASIS breast cancer cohorts exhibited hsa-mir-145-5p, hsa-mir-29a$3 p$, hsa-mir-17-3p, hsa-mir-17-5p, hsa-mir-18-5p, and hsa-mir-20a-5p as recurrently predicted breast cancer-associated miRNAs linked to cis-regulatory mutations and dysregulation of their target gene networks. Functional enrichment analysis confirmed that the dysregulated miRNA target gene networks are enriched for genes involved in transcriptional regulation and in cancerrelevant pathways such as the P53 pathway, hypoxia, and DNA damage response (Figure S26).

Finally, we further evaluated the clinical potential of the predicted breast cancer miRNAs for breast cancer survival estimation. For this purpose, we considered a third cohort, METABRIC [65], which is composed of 1282 samples. We computed Kaplan-Meier survival curves and log-rank tests using miRNA expression from the METABRIC cohort for the miRNAs predicted as drivers in the BRCA-US and BASIS cohort (for 24 of the predicted miRNAs in breast cancer). Examining both overall survival and breast cancer specific survival values, we observed significant log-rank test p-values for hsa-mir-29a-3p, hsa-mir-1290, and hsa-mir-20a$5 p$ (without multiple hypothesis correction; Figure 7 and Figures S27-S28). Note that hsa-mir20a-5p and hsa-mir-29a-3p were recurrently predicted in our analyses of the BRCA-US and BASIS cohorts. Taken together, these results reinforce a posteriori the potential of some miRNAs we predicted as their level of expression could be used for prognosis.
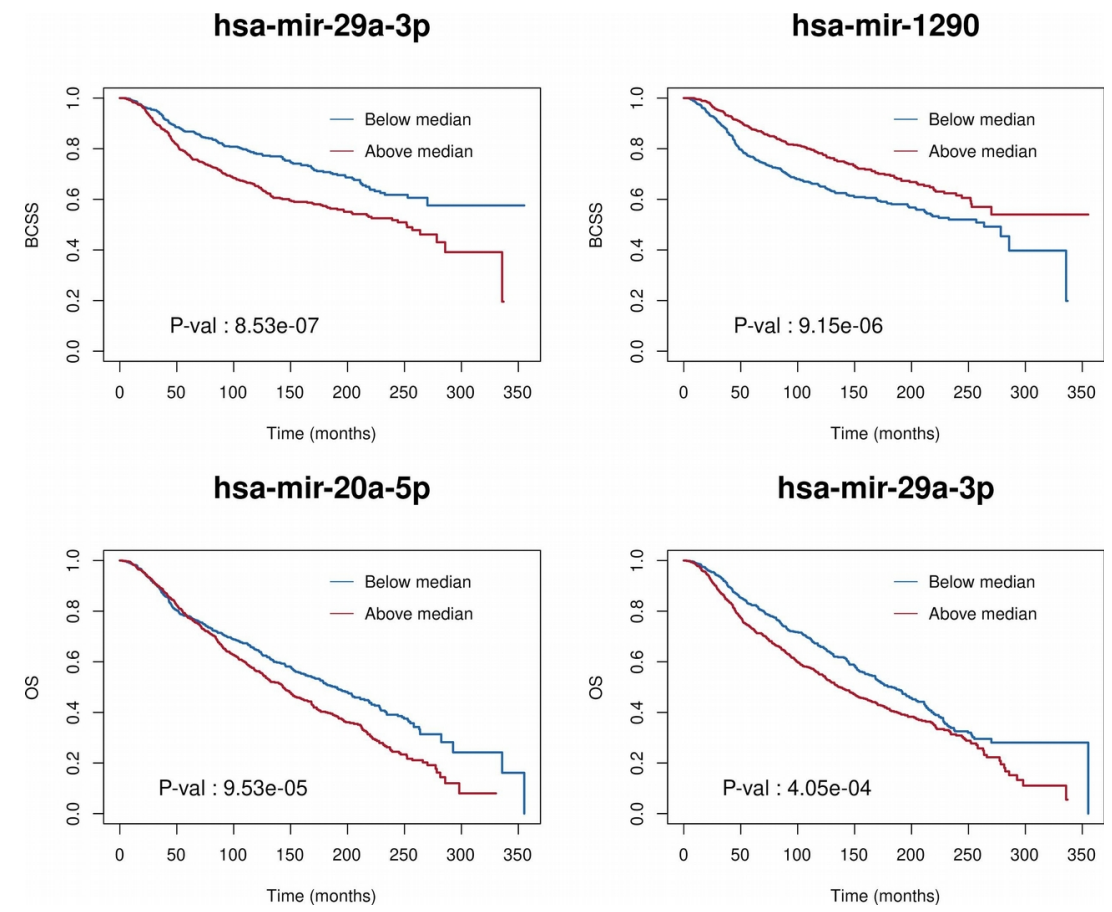

Figure 7. Survival curve analysis for some predicted miRNA drivers. Kaplan-Meier survival curves obtained using the METABRIC cohort for the most significant driver miRNAs identified in the breast cancer cohorts. Samples were separated into two groups according to the level of miRNA expression (above/below the median). Log-rank test $p$ values are indicated. OS: overall survival. BCSS: breast cancer specific survival. 


\section{DISCUSSION}

In this study, we explored how cis-regulatory mutations at TFBSs could be used to predict genes through the association of somatic mutations with a cascading trans-effect on gene regulatory network dysregulation. Contrary to most methods predicting cancer-driving events based on recurrence of mutations, we sought to couple cis-regulatory mutation information with gene expression data from the same samples to highlight direct evidence of the regulatory impact of the mutations. By integrating whole-genome somatic mutations, RNA-seq, and small RNA-seq data with gene regulatory networks, we performed pan-cancer predictions of proteincoding and miRNA genes associated with somatic cis-regulatory mutations in patients from seven distinct cancer types. Our study provides a large-scale foray to predict cancer-associated protein-coding and miRNA genes associated with somatic mutations by combining both transcriptional and post-transcriptional information. Our results provide new insights into the potential impacts and causes of the alteration of the gene regulatory program observed in cancer cells along with their cascading effects on key biological pathways.

We specifically focused on somatic mutations lying within a high-quality set of TFBSs representing direct TF-DNA interactions with both experimental and computational evidence [37] and covering $\sim 2 \%$ of the human genome. We acknowledge that this set of TFBSs might represent a limited subset of all potential TFBSs in the human genome as it was derived from experiments available for a reduced number of TFs and cell types/tissues (231 TFs out of the $\sim 1,600$ human TFs reported [11] and 315 cell types and tissues). Moreover, some TFBSs might not be active in the cell type of origin associated with the cancer types studied here. Nevertheless, we provided evidence that the regions considered are likely enriched for functional genomic elements since they harbour mutation rates similar to what is observed in exonic regions (Figure 1). The reduced mutation rates in exons and the limited increase in surrounding regions could be attributed to increased mismatch repair and nucleotide excision repair in exons as previously shown [66,67]. Our similar observation when considering our set of TFBSs is in line with our previous observation in B-cell lymphomas [27] but somewhat in disagreement with previous studies showing that nucleotide excision repair is impaired by the binding of TFs to DNA $[68,69]$. We hypothesize that the differences observed could be partially explained by the fact that (1) our mutation rate analysis considered TFBSs predicted from several cell lines and tissues instead of focusing on TFs and TFBSs specific to the considered cell types or conditions (such as UV-exposure in melanoma), and (2) we did not filter TFBSs based on open chromatin data from matched cell types.

Contrary to previous studies assessing the impact of mutations on TF-DNA binding affinity or the enrichment for mutations in cis-regulatory regions [70-72], we particularly evaluated the impact of cis-regulatory mutations on expression alteration in gene networks. As such, our approach does not quantify the direct impact of mutations on the obliteration of TF-DNA interactions but uses RNA information as the ultimate readout. A previous method systematically assessed the potential impact of somatic mutations in genomic tiles near genes' TSSs on gene expression [25]. Here, we considered mutations lying within a specific set of TFBSs without restrictions on distances to TSSs and evaluated the trans-association of the mutations with genes' network deregulation. Nevertheless, we acknowledge that the method misses mutations that would create new TFBSs as it is restricted to a set of predefined TFBSs. 
The analysis of protein coding genes showed 31 genes that were predicted in at least two (out of the seven) TCGA cohorts analyzed, with many already known cancer drivers (Figure 2A). We observed that the predicted protein-coding driver genes through the analysis of cis-regulatory mutations did generally not contain mutations in exonic regions for the same patients (Figures 2B and S13). This observation suggests complementary mechanisms acting upon gene expression dysregulation with cascading effect on regulatory network disruption.

Given that miRNAs cover a small portion of the human genome, they harbour a small number of somatic mutations [8], limiting the possibility to affect gene expression. The potential mechanism that we propose here is the alteration of their regulatory elements. Our study highlighted cis-regulatory mutations linked to miRNAs that were associated with dysregulation of expression of the miRNA targets. In our pan-cancer analysis, we discovered a core set of 17 miRNAs associated with the dysregulation of key pathways involved in carcinogenesis. This core set of miRNAs could represent a common feature for gene expression dysregulation associated with cancer onset or progression

The analysis of the dysregulated networks associated with the predicted cancer-associated genes (protein-coding and miRNAs) shows that many genes are dysregulated in a few samples but rarely across all the mutated samples (Figure 5B). This observation suggests a phenotypic heterogeneity (i.e. alterations of different parts of the same network lead to the same phenotype). However, the functional enrichment analysis of the dysregulated genes shows consistency across cohorts and across the analyzed types of mutations (LoF and cis-regulatory) for both protein-coding and miRNA genes. Moreover, as originally described by Ding et al. [26], the xseq probabilistic framework can highlight the specific samples where mutations are associated with impact on gene expression, while it does not in other samples (Figure 4A). This dichotomy can in principle be used to stratify samples and mutations but, in this study, was limited by the number of samples considered.

We applied our methodology to two cohorts of breast cancer samples (TCGA BRCA-US and BASIS). Given the large number of samples in BASIS $(n=256)$, we performed three analyses separately by considering (i) all samples, (ii) ER+ samples, and (iii) ER-samples. As expected, we observed that predictions can vary depending on the samples histopathology. This is particularly important for methods assessing impact on gene expression, which is influenced by the clinical composition of the cohorts. We acknowledge that methodological differences between the TCGA and BASIS cohorts (e.g. somatic mutation callings, small RNA-seq versus microarrays and normalization for miRNAs) could provide additional explanations for the variation in predictions. Although only one of the predicted protein-coding genes was predicted in both the BASIS and the BRCA-US cohorts, the functional enrichment analysis of the dysregulated gene networks was consistent. This observation suggests common dysregulated pathways acting as attractors that could originate from (non-recurrent) distinct cancerassociated events. It underlines the importance of addressing cancer as a disease with perturbations manifested at the gene network level. However, our miRNA analyses highlighted five miRNAs associated with cis-regulatory mutations and target gene expression alteration recurrently altered across the BRCA-US and BASIS breast cancer cohorts (Figure 6).

Despite the multiple lines of evidence for the prediction of cancer-associated genes in this 
study, we acknowledge that the predictions can provide false positives and false negatives due to multiple reasons such as: (i) a limited number of TFs with high-quality TFBSs; (ii) TFBS-target gene associations obtained by a naive approach combining information from an integrative database [39] and association to the closest TSS (Figure S8); we hypothesize that many of these associations may be irrelevant or incorrect and many others are missing; (iii) a diversity of tumor purity within the considered samples (despite the original threshold of $80 \%$ used by TCGA); (iv) a limited number of WGS datasets (tens of samples) within each cohort, compared to the number of samples with WXS (hundreds) used in other studies; (v) prior networks that might be incomplete. However, one of the main limitations of this project is the low number of tumor samples with both WGS and RNA-seq data, this limitation not only biases the community research towards the study of exonic regions, but also limits the statistical power of the methods assessing the impact of cis-regulatory mutations on gene network expression alteration.

Altogether, we argue that our capacity to predict cancer-associated cis-regulation mutations will increase as more high-quality TFBSs for more TFs and improved methods to associate TFBSs to their target genes become available. In addition, focusing on cis-regulatory regions specifically open or active in cancer samples would inform where somatic mutations are likely effective. We expect that with more WGS, RNA-seq, and other genomics datasets derived from cancer samples available, the community will resume the paucity in the detection of non-coding cancer-associated events [8].

\section{CONCLUSION}

By integrating whole-genome somatic mutations, RNA-seq, and small RNA-seq data with gene regulatory networks across seven cancer types, we were able to highlight cis-regulatory mutations associated with the dysregulation of gene regulatory networks through specific protein-coding and miRNA genes. The enrichment for known cancer genes, TFs, and the functional enrichment analysis reinforce a posteriori the predicted protein-coding and miRNA genes as being involved in biological pathway alteration affecting cancer development through exonic and cis-regulatory alterations. Our study represents, to our knowledge, the first largescale analysis of cis-regulatory mutations that are linked to gene expression alteration in key cancer-associated pathways. Our results suggest that this process can be achieved in a flexible way as we observed different genes in different patients but all associated with deregulation of the same pathways. Combining transcriptional and post-transcriptional information, we identified a core set of 17 miRNAs linked to altered cancer pathways across cancer types. These pancancer results here provide new insights into the impact and potential causes of miRNAmediated gene expression dysregulation. This work extends our capacity to address the discovery gap of cancer-associated events identification through the analysis of noncoding mutations and miRNA genes.

\section{MATERIAL AND METHODS}

All analyses were performed using the hg19 human genome assembly. When data was obtained from another human genome assembly, coordinates were converted to the hg19 assembly using the liftOver tool provided by the UCSC Genome Browser $[73,74]$. 


\section{Cancer patient data}

We considered TCGA [38] cohort samples for which trios of (i) whole genome somatic mutations, (ii) RNA-seq, and (iii) small RNA-seq data were available with at least 30 patients per cohort. Data was downloaded from the International Cancer Genome Consortium (ICGC) portal [75] through the icgc-get client (Additional file 6). Altogether, we collected data for 349 samples from seven TCGA patient cohorts (35 to 92 donors per cohort; Additional file 1): BRCAUS (breast invasive carcinoma), HNSC-US (head and neck squamous cell carcinoma), LIHCUS (liver hepatocellular carcinoma), LUAD-US (lung adenocarcinoma), LUSC-US (lung squamous cell carcinoma), STAD-US (stomach adenocarcinoma), and UCEC-US (uterine corpus endometrial carcinoma).

We collected data from 256 samples from the Breast Cancer Somatic Genetics Study (BASIS) cohort $[64,76]$ for which trios of whole genome somatic mutations, RNA-seq, and miRNA microarray data were available (Additional file 5). miRNA expression was measured using the Human miRNA Microarray Slide (Release 19.0) with Design ID 046064 (Agilent Technologies, Santa Clara, CA, USA; see ref. [64] for details).

\section{Somatic mutations}

Somatic single nucleotide variants (SNVs) and small insertions and deletions (indels) called by the tool MuSE [77] were retrieved from the ICGC portal for TCGA samples. For BASIS samples, we retrieved SNVs and indels called by the tools CaVEMan [78] and Pindel [79], respectively, used in the original study [64].

\section{RNA-seq and small RNA-seq normalization}

Both RNA-seq and small RNA-seq raw counts were filtered to remove all genes with 0 reads in more than $50 \%$ of the samples for a given cohort. For each cohort, both matrices (RNA-seq and small RNA-seq) of raw counts were normalized to counts per million (cpm) using the cpm function from the $\mathrm{R}$ package edgeR [80] and the cpm values were scaled by log 2 conversion. To avoid zeros, we added a pseudo-count of 1 . Note that small RNA-seq reads were mapped to pre-miRNA coordinates by TCGA, providing information about pre-miRNA expression and not mature miRNAs.

The normalized microarray miRNA expression matrix for BASIS samples was retrieved from the original study where normalization was performed using the 90th percentile methodology [64].

\section{Copy number alteration computation}

We downloaded copy number alteration (CNA) values predicted using the GISTIC2 tool [81] for TCGA samples through the Firebrowse database at http://firebrowse.org (Additional file 6). BASIS CNA estimates were computed using ASCAT (v2.1.1) [82] and converted into GISTIC format with -2 for homozygous loss (nMinor+nMajor=0), -1 for hemizygous loss (nMinor+nMajor=1), 0 for normal (nMinor+nMajor=2), 1 for three copies (nMinor+nMajor=3), and 2 for more than three copies (nMinor+nMajor>3). The CNA values assigned to the proteincoding genes were used in the $x$ seq analysis to remove cis-effects of CNAs on the gene 
expression dysregulation assessment [26].

\section{Mutation rate analysis}

For each sample, we calculated the mutation rates by dividing the total number of mutated nucleotides within a set of regions (TFBSs, exons, and flanking regions) by the total number of nucleotides covered by the given set of regions. TFBS genomic positions were obtained from UniBind [37] (see below). Protein-coding exon coordinates were retrieved from RefSeq Curated [83] (Additional file 6). Flanking regions were computed by (i) extending TFBS or exonic regions by 100,500 , and 1000 nucleotides on both sides using the flank bedtools subcommand and (ii) removing regions overlapping TFBSs and exonic regions using the subtract bedtools subcommand. Sets of regions were independently merged using the merge subcommand of the bedtools [84].

Random expectation for mutation rates were computed using 150 random sets of somatic mutations and applying the mutation rate computation described above. The random sets of mutations were generated by shuffling the original coordinates within the same chromosomes using the shuffle subcommand of the bedtools with the -chrom option.

\section{miRNAs}

Genomic coordinates of human pre-miRNAs were retrieved from miRBase v20 [45] as used to predict miRNA TSSs from CAGE data by the FANTOM5 consortium [36]. When miRNA names in the miRNA-related files (expression, survival, cancer-associated miRNAs) used in this study were mapped to older versions of miRBase (starting from version v10), we updated the names to the ones used in the latest miRBase version (v22) using the miRBaseConverter R/Bioconductor package [85].

\section{Transcription factor binding sites}

TFBSs were retrieved from the UniBind database (2019 version) at https://unibind.uio.no [37] (Additional file 6). The TFBSs correspond to high confidence direct TF-DNA interactions with both experimental (through ChIP-seq) and computational (through position weight matrices from JASPAR [86]) evidence. TFBSs were derived from 1983 ChIP-seq experiments for 231 TFs across 315 cell types and tissues [37].

\section{TFBS-gene association}

We used the cis-regulatory element-gene associations from the GeneHancer database (v4.9), derived from 8 sources to associate TFBSs to genes (Additional file 6; Figure S8) [39]. TFBSs overlapping a cis-regulatory element annotated in GeneHancer were associated with the corresponding gene in GeneHancer. TFBSs not overlapping annotated elements were associated with the closest TSS (for a protein-coding or a miRNA gene). We considered TSSs associated with protein-coding genes from RefSeq Curated [83] and TSSs associated with miRNAs by FANTOM5 [36]. With this approach, about half of the TFBSs were associated to protein-coding or miRNA genes using GeneHancer associations and the other half to the 
closest TSS.

\section{TFBS mutations}

Somatic mutations were intersected with TFBS locations using the intersect subcommand of bedtools v2.25.0 [84]. All mutations in TFBSs associated with miRNAs were considered for the xseq analysis (see below). For mutations in TFBSs associated with protein-coding genes, we followed the approach previously used by Mathelier et al. for the xseq analysis [27]. Specifically, we restricted the analysis to mutations associated with genes potentially dysregulated in the corresponding samples. Following ref. [27], genes were considered as potentially dysregulated in a given sample in cohort $C$ if its expression value $v$ satisfied $v<\mu-1 \sigma$ or $v>\mu+1 \sigma$ where $\mu$ and $\sigma$ correspond to the mean and standard deviation of the expression values of the gene in $C$.

\section{Loss-of-function mutations}

Following Ding et al. [26] for protein-coding exonic regions, we considered only LoF mutations that are either (i) nonsense mutations (disruptive in-frame deletion, disruptive in-frame insertion, stop gained, start lost, stop lost, and stop retained variant), (ii) frameshift mutations (frameshift variant, initiator codon variant), or (iii) splice-site mutations (splice region variant, splice donor variant, splice acceptor variant). The analysis was performed using somatic mutation data obtained from whole exon sequencing in the same TCGA samples as for the other analyses.

\section{Gene networks}

Protein-coding gene networks were retrieved from ref. [26] and were composed of 898,032 interactions. Briefly, the networks were constructed by combining gene associations from STRING v9.1 functional protein association [87], KEGG pathway datasets [40], WikiPathway [41], and BioCyc [88] as integrated in the IntPath database [89], and TF-target links from ENCODE [90] (see ref. [26] for more details). We updated the weights of the connections whenever possible using the methods provided in xseq, following the methodology described in ref. [26]. Specifically, the weight between a given gene $g$ and a biological partner gene $p$ was set to 1 if $p$ was found differentially expressed (Benjamini-Hochberg adjusted $p$ value $\leq 0.05$ ) in samples where $g$ is mutated in the same cohort (see Material and methods in ref. [26] for details). If there existed such genes $p$, then only these genes were kept connected to $g$. Original weights were kept otherwise.

\section{miRNA-target networks}

miRNAs were associated with potential target protein-coding genes using predictions from TargetScan v7.2 [31]. From the list of targets for each miRNA, we filtered out the targets with less than two predicted binding sites for the given miRNA to reduce false positives $[46,47]$. miRNA-target weights were computed as $t$ score / 100 where $t$ score corresponds to the targetScan context++ score percentiles from TargetScan. We updated the weights of the connections whenever possible following the same strategy as for protein-coding genes (see above). 


\section{xseq analyses}

The likely associations between mutations and dysregulation of gene or miRNA target networks were calculated with $x$ seq [26]. This method requires as input: a gene expression matrix of samples (RNA-seq matrix), a binary sample-gene mutation matrix, and a weighted network of connected genes. It outputs posterior probabilities associated to: (i) a sample-specific gene regulatory status (GRS, the probability of a given gene being dysregulated in a sample) for each gene connected to the gene associated with a mutation in a given sample, (ii) a sample-specific dysregulation probability (SSD, the probability that a mutation in a given gene in a given sample is associated with dysregulation of the gene's network), and (iii) a dysregulation across the cohort probability (DAC, the probability that mutations in a gene are associated with the dysregulation of its network across patients) (Figure S9). In a first step, we removed lowly expressed genes in a cohort following the approach described by Ding et al. [26]. Briefly, xseq considers the 90th percentile of expression for each gene and decomposes the distribution of these values into two Gaussian distributions corresponding to low and high expression values. We considered for further analysis the genes for which their 90th percentile of expression values lie within the high expression distribution with a posterior probability $\geq 0.8$ (see Ding et al. [26] for details). Next, xseq was used to compute all the posterior probabilities to predict genes and cis-regulatory mutations in the cancer patient cohorts. We considered potential cancerassociated genes the ones with $\mathrm{DAC} \geq 0.8$ and $\mathrm{SSD} \geq 0.5$ in at least two samples.

\section{Dysregulation heatmaps}

The dysregulated networks for predicted protein-coding and miRNA genes are visualized as heatmaps where columns correspond to mutated samples and rows to connected genes. Heatmaps were constructed with connected genes dysregulated (GRS $\geq 0.5$ ) in at least one sample with $S S D \geq 0.5$. These genes are referred to as dysregulated genes.

\section{Aggregated and sample-specific networks}

To evaluate whether the protein-coding genes predicted by cis-regulatory mutations are connected in the filtered networks (see Gene networks section), we built an aggregate network using all the predicted protein-coding genes within a cohort. We counted the number of disconnected subgraphs using the $\mathrm{R}$ packages igraph [91] and ggnetwork [92]. Similarly, we built sample-specific networks and counted the number of subgraphs in each sample, only considering the predicted genes with $\mathrm{DAC} \geq 0.8$ and $\mathrm{SSD} \geq 0.5$.

\section{Functional enrichment analysis}

Given a list of dysregulated genes, functional enrichment analyses were performed using the $\mathrm{R}$ package enrichR [44] for the following databases: KEGG_2019_Human, WikiPathways_2019_Human, GO_Biological_Process_2018, and Panther_2016.

\section{Enrichment for cancer genes and TFs}

Given a set of genes, we assessed their enrichment for cancer genes or TFs using 
hypergeometric tests using the stats::phyper function in R. The list of cancer protein-coding genes considered was constructed by considering genes that appear in at least two of the following databases: Network Cancer Gene [93], inToGen [94], and Cancer Gene Census [95]. Cancer miRNA genes were retrieved from miRCancer [48] (with data from May 1st 2019). TF genes were retrieved from the human transcription factor database [11].

\section{Survival analysis}

To test whether miRNA expression was associated with survival, we used the METABRIC breast cancer cohort [65] with miRNA microarray expression [96] available for 1282 tumors. Expression values were downloaded from the European Genome-Phenome Archive, www.ebi.ac.uk/ega, accession number EGAS00000000122. Follow-up data were available from Curtis et al. [65]. Kaplan-Meier survival analyses and log-rank tests were performed using the $\mathrm{R}$ package survival with tumors separated into "high" or "low" miRNA expression groups depending on expression values above or below the median.

\section{Results accessibility}

The analysis with all the scripts and parameters can be found through the following link: https://bitbucket.org/CBGR/workspace/projects/DYS. We provide (i) the source code for the analysis at https://bitbucket.org/CBGR/dysmir_manuscript/src/master/ and (ii) a pipeline for users to run similar analysis with their own data at https://bitbucket.org/CBGR/dysmir_pipeline/src/master/.

\section{SUPPLEMENTARY DATA}

Additional file 1. TCGA selected samples (specimen) with their ICGC/TCGA IDs. Samples were selected to provide WGS, RNA-seq, small RNA-seq, and CNA data. All samples considered in this study correspond to solid tumors.

Additional file 2. Summary of the number of mutations in the TCGA cohorts. Basic summary statistics (median, mean, and standard deviation) of the total number of mutations and the number of mutations overlapping TFBSs.

Additional file 3. Identified driver genes (miRNAs and protein coding genes) with their dysregulated targets.

Additional file 4. Summary of number of identified drivers and the number of dysregulated genes per cohort.

Additional file 5. BASIS selected samples and their ER status. Samples were selected to provide WGS, RNA-seq, miRNA microarray expression, and CNA data.

Additional file 6. Description of the resources used for this manuscript. Brief description of the resources (gene lists, databases, external files) used to perform this study, with links to their corresponding sites and the date when they were downloaded. 


\section{ACKNOWLEDGMENTS}

As research parasites [97], we thank the ICGC and BASIS consortia and other researchers for making their data publicly available. We thank Jiarui Ding for his help on using xseq for this study; Marcel Smid for providing raw RNA-seq data for the BASIS cohort; Georgios Magklaras and Georgios Marselis for their IT support; Ingrid Kjelsvik and Elisa Bjørgo for administrative support; Rafael Riudavets Puig, Roza Berhanu Lemma, Sebastian Waszak, Marieke Kuijjer, and Yuvia A. Perez-Rico for helpful comments on the manuscript; and the Mathelier and Kristensen's groups for insightful discussions throughout the course of execution of this project.

\section{FUNDING}

Norwegian Research Council [187615]; Helse Sør-Øst; University of Oslo through the Centre for Molecular Medicine Norway (NCMM) (to AM and JACM); Norwegian Research Council [288404 to JACM and Mathelier group]; The Norwegian Cancer Society [197884 to Mathelier group]. MRA was a postdoctoral fellow of the South Eastern Norway Health Authority (grant 2014021 to ALBD) and a research fellow of the Norwegian Cancer Society (grant 711164 to VNK).

Conflict of interest statement. None declared.

\section{CONTRIBUTION}

AM was responsible for the project conception and supervision. JACM was responsible for the analysis design and execution, and for its implementation. JACM and MRA undertook bioinformatic analysis. JACM, MRA, and AM wrote the manuscript with input from all co-authors. OCL contributed with CNA values for the BASIS cohort. JWMM contributed with clinical data. AL managed samples and clinical data. AM, JACM, MRA, ALBD, and VK contributed to the data analysis and scientific input. All authors read and approved the final manuscript.

\section{REFERENCES}

1. Bradner JE, Hnisz D, Young RA. Transcriptional Addiction in Cancer. Cell. 2017;168:629-43. 2. Melo SA, Esteller M. Dysregulation of microRNAs in cancer: playing with fire. FEBS Lett. 2011;585:2087-99.

3. Khurana E, Fu Y, Chakravarty D, Demichelis F, Rubin MA, Gerstein M. Role of non-coding sequence variants in cancer. Nat. Rev. Genet. 2016;17:93-108.

4. Cuykendall TN, Rubin MA, Khurana E. Non-coding genetic variation in cancer. Curr Opin Syst Biol. 2017;1:9-15.

5. Mathelier A, Shi W, Wasserman WW. Identification of altered cis-regulatory elements in human disease. Trends Genet. 2015;31:67-76.

6. Patel MB, Wang J. The Identification and Interpretation of cis-Regulatory Noncoding Mutations in Cancer. High Throughput. 2018;8.

7. Gan KA, Carrasco Pro S, Sewell JA, Fuxman Bass JI. Identification of Single Nucleotide Noncoding Driver Mutations in Cancer. Front Genet. 2018;9:16.

8. Rheinbay E, Nielsen MM, Abascal F, Wala JA, Shapira O, Tiao G, et al. Analyses of noncoding somatic drivers in 2,658 cancer whole genomes. Nature. 2020;578:102-11.

9. Kumar S, Warrell J, Li S, McGillivray PD, Meyerson W, Salichos L, et al. Passenger 
Mutations in More Than 2,500 Cancer Genomes: Overall Molecular Functional Impact and Consequences. Cell. 2020;180:915-27.e16.

10. Wasserman WW, Sandelin A. Applied bioinformatics for the identification of regulatory elements. Nat Rev Genet. 2004;5:276-87.

11. Lambert SA, Jolma A, Campitelli LF, Das PK, Yin Y, Albu M, et al. The Human Transcription Factors. Cell. 2018;172:650-65.

12. Weinhold N, Jacobsen A, Schultz N, Sander C, Lee W. Genome-wide analysis of noncoding regulatory mutations in cancer. Nat Genet. 2014;46:1160-5.

13. Fujimoto A, Furuta M, Totoki $Y$, Tsunoda T, Kato M, Shiraishi $Y$, et al. Whole-genome mutational landscape and characterization of noncoding and structural mutations in liver cancer. Nat Genet. 2016;48:500-9.

14. Lochovsky L, Zhang J, Fu Y, Khurana E, Gerstein M. LARVA: an integrative framework for large-scale analysis of recurrent variants in noncoding annotations. Nucleic Acids Res.

2015;43:8123-34.

15. Lochovsky L, Zhang J, Gerstein M. MOAT: efficient detection of highly mutated regions with the Mutations Overburdening Annotations Tool. Bioinformatics. 2018;34:1031-3.

16. Rheinbay E, Parasuraman P, Grimsby J, Tiao G, Engreitz JM, Kim J, et al. Recurrent and functional regulatory mutations in breast cancer. Nature. 2017;547:55-60.

17. Feigin ME, Garvin T, Bailey P, Waddell N, Chang DK, Kelley DR, et al. Recurrent noncoding regulatory mutations in pancreatic ductal adenocarcinoma. Nat Genet. 2017;49:825-33.

18. Shuai S, PCAWG Drivers and Functional Interpretation Working Group, Gallinger S, Stein L, PCAWG Consortium. Combined burden and functional impact tests for cancer driver discovery using DriverPower. Nat Commun. 2020;11:734.

19. Kalender Atak Z, Imrichova H, Svetlichnyy D, Hulselmans G, Christiaens V, Reumers J, et al. Identification of cis-regulatory mutations generating de novo edges in personalized cancer gene regulatory networks. Genome Med. 2017;9:80.

20. Fu Y, Liu Z, Lou S, Bedford J, Mu XJ, Yip KY, et al. FunSeq2: a framework for prioritizing noncoding regulatory variants in cancer. Genome Biol. 2014;15:480.

21. Ritchie GRS, Dunham I, Zeggini E, Flicek P. Functional annotation of noncoding sequence variants. Nat Methods. 2014;11:294-6.

22. Boyle AP, Hong EL, Hariharan M, Cheng Y, Schaub MA, Kasowski M, et al. Annotation of functional variation in personal genomes using RegulomeDB. Genome Res. 2012;22:1790-7. 23. Przytycki PF, Singh M. Differential Allele-Specific Expression Uncovers Breast Cancer Genes Dysregulated by Cis Noncoding Mutations. Cell Syst. 2020;10:193-203.e4.

24. Cheng Z, Vermeulen M, Rollins-Green M, DeVeale B. A catalog of cis-regulatory mutations in 12 major cancer types. bioRxiv 2019.

25. Fredriksson NJ, Ny L, Nilsson JA, Larsson E. Systematic analysis of noncoding somatic mutations and gene expression alterations across 14 tumor types. Nat Genet. 2014;46:125863.

26. Ding J, McConechy MK, Horlings HM, Ha G, Chun Chan F, Funnell T, et al. Systematic analysis of somatic mutations impacting gene expression in 12 tumour types. Nat Commun. 2015;6:8554.

27. Mathelier A, Lefebvre C, Zhang AW, Arenillas DJ, Ding J, Wasserman WW, et al. Cisregulatory somatic mutations and gene-expression alteration in B-cell lymphomas. Genome Biol. 2015;16:84.

28. Bartel DP, Chen C-Z. Micromanagers of gene expression: the potentially widespread influence of metazoan microRNAs. Nat Rev Genet. 2004;5:396-400. 
29. Bartel DP. Metazoan MicroRNAs. Cell. 2018;173:20-51.

30. Ha M, Kim VN. Regulation of microRNA biogenesis. Nat Rev Mol Cell Biol. 2014;15:509-24.

31. Agarwal V, Bell GW, Nam J-W, Bartel DP. Predicting effective microRNA target sites in mammalian mRNAs. Elife 2015;4.

32. Brancati G, Großhans H. An interplay of miRNA abundance and target site architecture determines miRNA activity and specificity. Nucleic Acids Res. 2018;46:3259-69.

33. Lin S, Gregory RI. MicroRNA biogenesis pathways in cancer. Nat Rev Cancer. 2015;15:321-33.

34. Peng Y, Croce CM. The role of MicroRNAs in human cancer. Signal Transduction and Targeted Therapy. 2016.

35. Kern F, Backes C, Hirsch P, Fehlmann T, Hart M, Meese E, et al. What's the target: understanding two decades of in silico microRNA-target prediction. Brief Bioinform. 2019.

36. de Rie D, Abugessaisa I, Alam T, Arner E, Arner P, Ashoor H, et al. An integrated expression atlas of miRNAs and their promoters in human and mouse. Nat Biotechnol. 2017;35:872-8.

37. Gheorghe M, Sandve GS, Khan A, Cheneby J, Ballester B, Mathelier A. A map of direct TFDNA interactions in the human genome. Nucleic Acids Research. 2018.

38. Weinstein JN, The Cancer Genome Atlas Research Network, Collisson EA, Mills GB, Mills Shaw KR, Ozenberger BA, et al. The Cancer Genome Atlas Pan-Cancer analysis project. Nature Genetics. 2013. p. 1113-20.

39. Fishilevich S, Nudel R, Rappaport N, Hadar R, Plaschkes I, Iny Stein T, et al. GeneHancer: genome-wide integration of enhancers and target genes in GeneCards. Database 2017.

40. Kanehisa M, Goto S. KEGG: kyoto encyclopedia of genes and genomes. Nucleic Acids Res. 2000;28:27-30.

41. Pico AR, Kelder T, van Iersel MP, Hanspers K, Conklin BR, Evelo C. WikiPathways: pathway editing for the people. PLoS Biol. 2008;6:e184.

42. Thomas PD, Campbell MJ, Kejariwal A, Mi H, Karlak B, Daverman R, et al. PANTHER: a library of protein families and subfamilies indexed by function. Genome Res. 2003;13:2129-41. 43. Ashburner M, Ball CA, Blake JA, Botstein D, Butler H, Cherry JM, et al. Gene ontology: tool for the unification of biology. The Gene Ontology Consortium. Nat Genet. 2000;25:25-9.

44. Kuleshov MV, Jones MR, Rouillard AD, Fernandez NF, Duan Q, Wang Z, et al. Enrichr: a comprehensive gene set enrichment analysis web server 2016 update. Nucleic Acids Res. 2016;44:W90-7.

45. Kozomara A, Griffiths-Jones S. miRBase: annotating high confidence microRNAs using deep sequencing data. Nucleic Acids Res. 2014;42:D68-73.

46. Seitz H. Issues in current microRNA target identification methods. RNA Biol. 2017;14:831-4. 47. Krek A, Grün D, Poy MN, Wolf R, Rosenberg L, Epstein EJ, et al. Combinatorial microRNA target predictions. Nat Genet. 2005;37:495-500.

48. Xie B, Ding Q, Han H, Wu D. miRCancer: a microRNA-cancer association database constructed by text mining on literature. Bioinformatics. Narnia; 2013;29:638-44.

49. Sayers EW, Barrett T, Benson DA, Bolton E, Bryant SH, Canese K, et al. Database resources of the National Center for Biotechnology Information. Nucleic Acids Res.

2011;39:D38-51.

50. Mendell JT. miRiad roles for the miR-17-92 cluster in development and disease. Cell. 2008;133:217-22.

51. Teng G, Papavasiliou FN. Shhh! Silencing by microRNA-155. Philos Trans R Soc Lond B 
Biol Sci. 2009;364:631-7.

52. Nadiminty N, Tummala R, Lou W, Zhu Y, Shi X-B, Zou JX, et al. MicroRNA let-7c is downregulated in prostate cancer and suppresses prostate cancer growth. PLoS One. 2012; 7:e32832.

53. Tehler D, Høyland-Kroghsbo NM, Lund AH. The miR-10 microRNA precursor family. RNA Biol. 2011;8:728-34.

54. Ke K, Lou T. MicroRNA-10a suppresses breast cancer progression via PI3K/Akt/mTOR pathway. Oncol Lett. 2017;14:5994-6000.

55. Mu N, Gu J, Huang T, Zhang C, Shu Z, Li M, et al. A novel NF-kB/YY1/microRNA-10a regulatory circuit in fibroblast-like synoviocytes regulates inflammation in rheumatoid arthritis. Sci Rep. 2016;6:20059.

56. Hirschberger S, Hinske LC, Kreth S. MiRNAs: dynamic regulators of immune cell functions in inflammation and cancer. Cancer Lett. 2018;431:11-21.

57. Kim E-A, Kim S-W, Nam J, Sung E-G, Song I-H, Kim J-Y, et al. Inhibition of c-FLIPL expression by miRNA-708 increases the sensitivity of renal cancer cells to anti-cancer drugs. Oncotarget. 2016;7:31832-46.

58. Lee J-W, Guan W, Han S, Hong D-K, Kim L-S, Kim H. MicroRNA-708-3p mediates metastasis and chemoresistance through inhibition of epithelial-to-mesenchymal transition in breast cancer. Cancer Sci. 2018;109:1404-13.

59. Li J, Li L, Li Z, Gong G, Chen P, Liu H, et al. The role of miR-205 in the VEGF-mediated promotion of human ovarian cancer cell invasion. Gynecol Oncol. 2015;137:125-33.

60. Tuo H, Wang Y, Wang L, Yao B, Li Q, Wang C, et al. MiR-324-3p promotes tumor growth through targeting DACT1 and activation of Wnt/ $\beta$-catenin pathway in hepatocellular carcinoma. Oncotarget. 2017;8:65687-98.

61. Lu J, Lu S, Li J, Yu Q, Liu L, Li Q. MiR-629-5p promotes colorectal cancer progression through targetting CXXC finger protein 4. Biosci Rep. 2018;38.

62. Karimzadeh M, Arlidge C, Rostami A, Lupien M, Bratman SV, Hoffman MM. Viral integration transforms chromatin to drive oncogenesis.

63. Zapatka M, Borozan I, Brewer DS, Iskar M, Grundhoff A, Alawi M, et al. The landscape of viral associations in human cancers. Nat. Genet. 2020.

64. Nik-Zainal S, Davies H, Staaf J, Ramakrishna M, Glodzik D, Zou X, et al. Landscape of somatic mutations in 560 breast cancer whole-genome sequences. Nature. 2016;534:47-54.

65. Curtis C, Shah SP, Chin S-F, Turashvili G, Rueda OM, Dunning MJ, et al. The genomic and transcriptomic architecture of 2,000 breast tumours reveals novel subgroups. Nature.

2012;486:346-52.

66. Martincorena I, Raine KM, Gerstung M, Dawson KJ, Haase K, Van Loo P, et al. Universal Patterns of Selection in Cancer and Somatic Tissues. Cell. 2017. p. 1029-41.e21.

67. Frigola J, Sabarinathan R, Mularoni L, Muiños F, Gonzalez-Perez A, López-Bigas N.

Reduced mutation rate in exons due to differential mismatch repair. Nat Genet. 2017;49:168492.

68. Sabarinathan R, Mularoni L, Deu-Pons J, Gonzalez-Perez A, López-Bigas N. Nucleotide excision repair is impaired by binding of transcription factors to DNA. Nature. 2016;532:264-7. 69. Kaiser VB, Taylor MS, Semple CA. Mutational Biases Drive Elevated Rates of Substitution at Regulatory Sites across Cancer Types. PLoS Genet. 2016;12:e1006207.

70. Nishizaki SS, Ng N, Dong S, Porter RS, Morterud C, Williams C, et al. Predicting the effects of SNPs on transcription factor binding affinity. Bioinformatics. 2020;36:364-72.

71. Fornes O, Gheorghe M, Richmond PA, Arenillas DJ, Wasserman WW, Mathelier A. 
MANTA2, update of the Mongo database for the analysis of transcription factor binding site alterations. Sci Data. 2018;5:180141.

72. Gonzalez-Perez A, Sabarinathan R, Lopez-Bigas N. Local Determinants of the Mutational Landscape of the Human Genome. Cell. 2019;177:101-14.

73. Hinrichs AS, Karolchik D, Baertsch R, Barber GP, Bejerano G, Clawson H, et al. The UCSC Genome Browser Database: update 2006. Nucleic Acids Res. 2006;34:D590-8.

74. Karolchik D, James Kent W. The UCSC Genome Browser. Current Protocols in Bioinformatics. 2003. p. 1.4.1-1.4.23.

75. Zhang J, Baran J, Cros A, Guberman JM, Haider S, Hsu J, et al. International Cancer Genome Consortium Data Portal-a one-stop shop for cancer genomics data. Database. 2011 76. Smid M, Rodríguez-González FG, Sieuwerts AM, Salgado R, Prager-Van der Smissen WJC, Vlugt-Daane $\mathrm{M}$ van der, et al. Breast cancer genome and transcriptome integration implicates specific mutational signatures with immune cell infiltration. Nat Commun. 2016;7:12910.

77. Fan Y, Xi L, Hughes DST, Zhang J, Zhang J, Futreal PA, et al. MuSE: accounting for tumor heterogeneity using a sample-specific error model improves sensitivity and specificity in mutation calling from sequencing data. Genome Biol. 2016;17:178.

78. Jones D, Raine KM, Davies H, Tarpey PS, Butler AP, Teague JW, et al. cgpCaVEManWrapper: Simple Execution of CaVEMan in Order to Detect Somatic Single Nucleotide Variants in NGS Data. Curr Protoc Bioinformatics. 2016;56:15.10.1-15.10.18.

79. Raine KM, Hinton J, Butler AP, Teague JW, Davies H, Tarpey P, et al. cgpPindel: Identifying Somatically Acquired Insertion and Deletion Events from Paired End Sequencing. Curr Protoc Bioinformatics. 2015;52:15.7.1-12.

80. Robinson MD, McCarthy DJ, Smyth GK. edgeR: a Bioconductor package for differential expression analysis of digital gene expression data. Bioinformatics. 2010;26:139-40.

81. Mermel CH, Schumacher SE, Hill B, Meyerson ML, Beroukhim R, Getz G. GISTIC2.0 facilitates sensitive and confident localization of the targets of focal somatic copy-number alteration in human cancers. Genome Biol. 2011;12:R41.

82. Van Loo P, Nordgard SH, Lingjærde OC, Russnes HG, Rye IH, Sun W, et al. Allele-specific copy number analysis of tumors. Proc Natl Acad Sci U S A. 2010;107:16910-5.

83. O'Leary NA, Wright MW, Brister JR, Ciufo S, Haddad D, McVeigh R, et al. Reference sequence (RefSeq) database at NCBI: current status, taxonomic expansion, and functional annotation. Nucleic Acids Res. 2016;44:D733-45.

84. Quinlan AR, Hall IM. BEDTools: a flexible suite of utilities for comparing genomic features. Bioinformatics. 2010;26:841-2.

85. Xu T, Su N, Liu L, Zhang J, Wang H, Zhang W, et al. miRBaseConverter: An

R/Bioconductor Package for Converting and Retrieving miRNA Name, Accession, Sequence and Family Information in Different Versions of miRBase.

86. Khan A, Fornes O, Stigliani A, Gheorghe M, Castro-Mondragon JA, van der Lee R, et al. JASPAR 2018: update of the open-access database of transcription factor binding profiles and its web framework. Nucleic Acids Res. 2018;46:D1284.

87. Franceschini A, Szklarczyk D, Frankild S, Kuhn M, Simonovic M, Roth A, et al. STRING v9.1: protein-protein interaction networks, with increased coverage and integration. Nucleic Acids Res. 2013;41:D808-15.

88. Karp PD, Ouzounis CA, Moore-Kochlacs C, Goldovsky L, Kaipa P, Ahrén D, et al. Expansion of the BioCyc collection of pathway/genome databases to 160 genomes. Nucleic Acids Res. 2005;33:6083-9. 
89. Zhou H, Jin J, Zhang H, Yi B, Wozniak M, Wong L. IntPath--an integrated pathway gene relationship database for model organisms and important pathogens. BMC Syst Biol. 2012;6 Suppl 2:S2.

90. Gerstein MB, Kundaje A, Hariharan M, Landt SG, Yan K-K, Cheng C, et al. Architecture of the human regulatory network derived from ENCODE data. Nature. 2012;489:91-100.

91. Csardi G, Nepusz T, Others. The igraph software package for complex network research. InterJournal, complex systems. 2006;1695:1-9.

92. Tyner S, Briatte F, Hofmann H. Network Visualization with ggplot2. The R Journal. 2017. p. 27.

93. Repana D, Nulsen J, Dressler L, Bortolomeazzi M, Venkata SK, Tourna A, et al. The Network of Cancer Genes (NCG): a comprehensive catalogue of known and candidate cancer genes from cancer sequencing screens. Genome Biol. 2019;20:1.

94. Gundem G, Perez-Llamas C, Jene-Sanz A, Kedzierska A, Islam A, Deu-Pons J, et al. IntOGen: integration and data mining of multidimensional oncogenomic data. Nat Methods. 2010;7:92-3.

95. Sondka Z, Bamford S, Cole CG, Ward SA, Dunham I, Forbes SA. The COSMIC Cancer Gene Census: describing genetic dysfunction across all human cancers. Nat Rev Cancer. 2018;18:696-705.

96. Dvinge H, Git A, Gräf S, Salmon-Divon M, Curtis C, Sottoriva A, et al. The shaping and functional consequences of the microRNA landscape in breast cancer. Nature. 2013. p. 378-82.

97. Longo DL, Drazen JM. Data Sharing. N. Engl. J. Med. 2016. p. 276-7. 